Mathias Verbeke*, Bettina Berendt, Leen d'Haenens and Michaël Opgenhaffen

\title{
Critical news reading with Twitter? Exploring data-mining practices and their impact on societal discourse
}

DOI 10.1515/commun-2017-0014

\begin{abstract}
This article shows that the collaboration between social science and computer science scholars proves fruitful in enhancing conceptual and methodological innovation in research appropriate for the digital world. It presents arguments for ways in which a multi-disciplinary approach can strengthen media studies and innovatively advance both research breadth and depth. To illustrate this interesting connection of both disciplines, we present the example analysis of large data from Twitter and discuss this analysis in a communication science research environment. We propose TwiNeR, a software tool that analyzes tweet content using an advanced language modeling approach for classifying tweets into five prototypical messages referring to 'activities' related to news and news sources in the Twitter network (i.e., source-fed article, userfed article, content spread by user, other source content, other user content).
\end{abstract}

Keywords: news analysis, Twitter analysis, big data, mixed media environment, multi-disciplinary approach

\section{Aims and scope}

Big data practices of social media are transforming the way many people interact with one another, show public engagement, and receive and produce information (Meraz, 2011a). In the fragmentized media climate, news media are now

\footnotetext{
*Corresponding author: Mathias Verbeke, Department of Computer Science, University of Leuven (KU Leuven), E-mail: Mathias.Verbeke@cs.kuleuven.be

Bettina Berendt, Department of Computer Science, University of Leuven (KU Leuven), E-mail: bettina.berendt@cs.kuleuven.be

Leen d'Haenens, Institute for Media Studies, University of Leuven (KU Leuven),

E-mail: leen.dhaenens@soc.kuleuven.be

Michaël Opgenhaffen, Institute for Media Studies, University of Leuven (KU Leuven), E-mail: michael.opgenhaffen@soc.kuleuven.be
} 
themselves actors in social media, establishing their own practices as well as developing practices of interaction and engagement with non-traditional sources such as citizens, who can opt to become creators or distributors of news as "citizens or accidental journalists" (Allan, 2013). Crucial in this respect are the ways in which traditional activities of news production, publication and dissemination unfold in this new ecosystem, and how the different actors' practices interact to shape public opinion.

We will focus on the microblogging system Twitter and on Twitter messages (tweets) relating to mainstream media. We do not investigate the contents of messages or of public opinion, but focus on the communication structure as such, particularly on who is active in distributing content and/or enriching content. We regard this as a proxy measure of how people read and process news, to what extent they engage in critical news reading, and thus ultimately of how they form and shape opinions.

Methodologically, we explore how the big data generated by the big data practices on social media can give us insights into those practices when analyzed by means of data mining as a big data research method. Furthermore, we want to use this example to demonstrate how issues arising in this process of interdisciplinary collaboration challenge and enrich the assumptions, questions and interpretations of both disciplines involved in this study: computer science (first and second author) and communication science (third and fourth author).

Through this combination of content and method questions, the article investigates big data viewed as a field of practices generative of data and analysis methods, and as influencing both society and research practices. Of course, within the scope of this article we can neither give an exhaustive account and analysis of 'news' on social media, nor address, let alone solve, all issues of interdisciplinary collaboration in this field. We do hope, by concentrating on a specific content question and giving a rather detailed account of the methodological challenges encountered and how we addressed them, to contribute to an understanding of the domain and spark further interdisciplinary research.

This article has two goals:

1. To reflect on how communication science and computer science can be instrumental for one another, informing each other on their respective conceptual worlds as well as on methodologies and tools, advancing both domains.

2. To prove this argument by way of an illustration. TwiNeR, a tool for visualization of different types of Twitter messages, is proposed, its core idea being the categorization of Twitter messages into one of the several types based on the type of news spreading activity it included. Such analysis, explorative as it is, will be instructive about the ways in which the Twitter network 
operates, whether Twitter users mostly filter for their followers by selecting and re-assembling interesting content 'as is', or whether they comment on what they see on the web. We will also find out whether these behaviors are affected by the origin of the first tweet-mainstream news source or some tweep, that is, the person who tweets.

\section{Related work}

\subsection{Big data analysis in communication science}

Through the rise of social media and the increasing digitization of the news media industry, social science researchers can avail themselves of a huge number of new knowledge sources. In addition to its obvious opportunities, social science research in 'internet times' also entails new challenges. Karpf (2012) outlines three potential problems. The first is that the underlying concepts are not immutable; they change while being studied. Second, these changes can have an impact on the ceteris paribus assumptions of social science research methods. Furthermore, gathering and analyzing publicly-available online data has a number of limitations. Applying novel techniques to such 'dirty' big data often causes problems, such as identifying fake spam accounts in social networks and dealing with the noise introduced by them.

John Naisbitt (1982) noted that "we are drowning in data, yet starving for knowledge". This quote aptly summarizes the bottleneck that communication science currently faces when investigating 'big data'. We define big data as massive volumes of both structured and unstructured information that is dynamic in nature, that is collected over a certain period of time, and that requires computational methods for extracting knowledge from it. As illustrated in numerous content analyses based on manual analysis and purposeful sampling, current research efforts in communication science have mostly concentrated on analyzing static content in a limited time frame. This is mainly because current techniques generally focus on one particular aspect of the data: either content (e.g., the topics of news articles) or network structure (e.g., the interconnections between interacting users on social networks). Such approaches neglect the dynamics within the network and the content on offer as well as the temporal contingency and updates of information, which are characteristic especially of data in the news research domain. In this article, we propose data mining as a possible solution to Karpf's challenges and the digital data overload we are facing today. Data mining is the computational process of discovering patterns 
(i.e., knowledge) in large data sets using methods which lie at the intersection of artificial intelligence, machine learning, statistics, and database systems. We present an overview of data mining methods for handling 'big data' in communication science, with a view to the empowerment of the communication scholar aiming to extract knowledge from 'big data'. As transforming the resulting data analysis into knowledge requires the necessary domain expertise of the communication scientist, this interdisciplinary approach can also drive research in computer science, in the domains of data and text mining, the processing of time-critical information, and visual analytics. Against the background of general methodological questions (e.g., How can conceptual worlds of computer science and communication science be instrumental to one another?) as well as more practical issues (e.g., Which API could one use?) related to the introduction of 'big data' in communication research, a case study of the Twitter network is presented. It is worth noting that the methodology in question also applies to other data sources, but that we use Twitter as an example of dynamic news sources that are currently studied in communication science. The case study will illustrate some of the many roles Twitter may fulfill; the active and passive uses for news handling (as a community forum or as a news ticker).

\subsection{Data mining for big data analysis in communication science}

Data mining is the non-trivial computational process of identifying valid, novel, potentially useful, and ultimately understandable patterns in large amounts of data (Fayyad, Piatestky-Shapiro, and Smyth, 1996). Data mining can be divided into several subfields, depending on the type of data that is mined (Witten, Frank, and Hall, 2011). Text and web mining are two of them, where the data consists of text and web data, respectively. Social-web mining is a hybrid domain, in which techniques from text and web mining and social network analysis are combined to mine social network data. Text and web mining tasks specifically meant for news analysis are discussed by Berendt (2010). For example, news data is indexed by time, it makes fewer assumptions about contextual knowledge than other texts, and the social structure present in discussions about news has an impact on the content and meaning. These news data characteristics lead to a number of new, specific research areas in the field (e.g., topic detection and tracking, see Allan, 2002).

The use of social media in news reporting is no longer uncommon, the London riots (Glasgow and Fink, 2013), or the real-time reporting throughout 
the Arab Spring (Papacharissi and de Fatima Oliveira, 2012) being prominent examples. In the case of the latter, the role of social media went beyond that of a news disseminator: Communication over Facebook, Twitter and others exerted a large influence on the course of events (e.g., Tufekci and Wilson, 2012). Social networks thus contain a wealth of information. Social network analysis (Easley and Kleinberg, 2010) studies the social relationships in these networks based on graph theory.

We can now define (social) news analysis as the study of news-related phenomena in (social) data by means of a combination of data mining methods. Examples are the use of text mining methods to identify rumors in social networks (Qazvinian, Rosengren, Radev, and Mei, 2011) and to contextualize news articles (Gamon et al., 2008). Temporal text mining (Mei and Zhai, 2005) adds the dimension of time, which has been extended to a graph-based approach to do news-story tracking (Subašić and Berendt, 2010). Other state-of-the-art methods in the context of news analysis include the intermedia agenda-setting influence through the measurement of issue or source salience, coherence or discrepancy between traditional media and newer media platforms (e.g., Meraz, 2011b); measuring user influence in social networks (Cha et al., 2010); and research done in the context of meme-tracking with applications to the news cycle (Leskovec, Backstrom, and Kleinberg, 2009), that is, studying the migration of short units of text across a group of people. We will now focus on the analysis of Twitter, as a particular instance of a social network and a big data source for research in communication studies.

\subsection{Twitter: Big data source, behavioral studies, and analysis tools}

Due to the underlying structure of the platform with its hashtag system and the ability to broadcast public messages, Twitter can support the ad hoc formation of large online communities debating different topics (Bruns and Burgess, 2012). When combined with the possibility to send short messages in real time to a network of followers - who can redistribute them further to their followers - Twitter provides for the quick dissemination of news and the voicing of public opinion. Hence the platform's irresistible attraction for journalists, companies, politicians and emergency services.

In October 2014, Twitter had roughly 271 million monthly active users, whose aggregate daily output averaged 500 million tweets. ${ }^{1}$ Due to this popu-

1 https://about.twitter.com/company 
larity, it has also become an active research subject in the social sciences and the humanities (e.g., sociology, political sciences, linguistics) and in computer science, mostly in the form of social network analysis. This has led to a large number of promising approaches in different research areas. Sample questions deal with how politicians use Twitter during election times (Vergeer and Hermans, 2013), or how brands and organizations interact with their audiences (Jothi, Neelamalar, and Prasad, 2011). Twitter also plays an important role in crisis events. Scholars have studied the use and impact of the platform as an emergency response tool, as in the cases of the N1H1-pandemic (Chew and Eysenbach, 2010). A detailed study investigating the possibilities of real-time and automated analysis of Twitter messages during crises can be found in Terpstra, de Vries, Stronkman, and Paradies (2012).

A collection of tweets is the ideal subject for the analysis of the frequency and percentage of themes or hashtags (e.g., Sullivan et al., 2012), the sentiment of tweets (e.g., Chew and Eysenbach, 2010), and the detection of emerging topics (Benhardus and Kalita, 2013). To analyze phenomena that change over time, time series analyses can be run and subsequently combined with sentiment analysis (e.g., Thelwall, Buckley and Paltoglou, 2011). Using social network analysis techniques, Bollen, Mao, and Pepe (2011) measure the relationship between sentiment in Twitter posts and socio-economic phenomena, and Kwak, Lee, Park, and Moon (2010) study the topological characteristics of Twitter and its power as a new medium of information sharing.

Various studies have investigated Twitter in relation to news, for instance, to study the speed at which news is spread (e.g., Lerman and Ghosh, 2010), or how news media refer to men and women in Twitter feeds and how this relates to portrayals in news stories (Armstrong and Gao, 2011). It has been shown that Twitter acts more as a source of information (most of which comes from headline news) than as a social medium (Kwak et al., 2010), and that in spite of the large opportunities it creates for citizen participation in the news-making process, a top-down approach starting with trusted news sources is still dominant (Hu et al., 2012; Szomszor, Kostkova, and St Louis, 2009). When Twitter users do not just spread news coming from mainstream news media, they tend to comment on these rather than spreading their own, 'different' news (Subašić and Berendt, 2011). News plays an important role in the conversations and collaborations that take place on Twitter (Hansen, Arvidsson, Nielsen, Colleoni, and Etter, 2011; Honey and Herring, 2009). Retweets are a central mechanism of becoming part of a conversation (Boyd, Golder, and Lotan, 2010).

Along with the increasing amount of research on Twitter, a number of tweet analysis tools have been presented. Many of these have been developed to give 
the user more insight into Twitter dynamics. Twitalyzer ${ }^{2}$ and Topsy ${ }^{3}$ provide social analytics tools that combine different measures with demographic data and help users get an idea of which of their efforts on social networks are effective. Twimpact ${ }^{4}$ provides a searchable real-time feed for retweet, image and link posting trends on different time scales.

Some of these tools have been specifically developed to support journalists in the reporting and publishing process. With 'Twitter for Newsrooms', 5 Twitter gathered and created a tailored set of tools to help journalists find sources, check facts, publish stories and promote their work. NewsWhip 6 tracks all the news published by about 5,000 English-language sources. For each news event, it collects the numbers of shares, likes, tweets and comments. Using this information, it calculates the social speed at which each news event is traveling, giving the user an idea of how popular a particular topic has become at a certain point in time. Based on this technology, two related tools have been developed. NewsWhip's Spike ${ }^{7}$ uses the gathered information to detect trending stories (i.e., a topic or event which is currently popular and much discussed online); Social Amplifier ${ }^{8}$ displays a news company's most trending stories in real time, and gives readers and journalists a live view of what the site's users are sharing. A set of methods for filtering and assessing the verity of sources found through social media in the case of breaking news events is presented by Diakopoulos, De Choudhury, and Naaman (2012).

Notwithstanding Twitter's simple and open communication model, the richness of the information provided and the interesting social network structure, analyzing Twitter data also presents a number of new challenges, such as the speed at which the data comes in and the different number of data formats we are faced with today. To the end, the methods and techniques from computer science come in helpful. At the same time, the experimental setup and interpretation of the resulting analysis also requires the necessary domain knowledge. As indicated by Boyd and Crawford (2012), interpretation is at the center of data analysis. Without a decent understanding of the limitations and biases of the data, misinterpretation is lurking, which is why the expertise of the communication scientist is required.

2 http://www.twitalyzer.com

3 http://www.topsy.com

$4 \mathrm{http} / / /$ www.twimpact.com

5 https://dev.twitter/com/media/newsrooms

$6 \mathrm{http}: / /$ www.newswhip.com

7 http://spike.newswhip.com

8 http://www.newswhip.com/SocialAmplifier 


\section{A model of news reading (step 1)}

In this and the following sections, we will present a model of news reading illustrating the general framework described. For ease of reading, we have aggregated across highly interdependent steps.

Step 1: From a communications-research question to a computational-research question

In our case study, we investigated how to use Twitter to study news reading behavior. To this end, we needed to dynamically combine multiple data sources (i.e., Twitter data with news articles) and applied techniques from data mining in order to find relevant patterns in the communication behavior of the different actors. With this in mind, we developed TwiNeR, a software tool to automatically process large numbers of tweets and visually inspect the results.

The goal is to help both researchers and news-content providers understand these large-scale phenomena and compare behaviors that relate to different news sources. This will make it possible, for example, to compare the involvement and types of activity of major news providers and their readers in Europe with those of others in Africa or elsewhere. Such quantitative descriptions can support the investigation of more comprehensive questions regarding the role of microblogging in shaping critical and outspoken news-reading audiences.

The basic idea of our model is a classification of tweets by their position in the information diffusion cycle. This cycle originates from people's reading, reception and reactions to reading news source content on the web and on Twitter. The result is shown as part of a potential activity model in Figure 1. The types in this model are explained in Table 1.

The types emphasize which actor (the news source or another user) tweets and also whether the tweet serves to introduce content into the Twitter network (no retweet) or to spread existing content in it (retweet).

A third characteristic of tweets that link to a webpage is their relation to this page: Does the tweet quote and/or add? In other words, does it re-report existing content from a webpage ${ }^{9}$, and/or does it contain the user's own words, which may be further factual content, opinions, or other forms of comment? Figure 1 illustrates this by showing the types of tweets with shading from 'white' (no user-generated content) to 'black' (only user-generated content).

9 We write "a" webpage because only very few tweets link to more than one page. 
Table 1: Types of tweets.

\begin{tabular}{|c|c|}
\hline $\begin{array}{l}\text { Source-fed } \\
\text { article content }\end{array}$ & The news source itself feeds a news item into the Twitter network. \\
\hline $\begin{array}{l}\text { User-fed } \\
\text { article content }\end{array}$ & $\begin{array}{l}\text { A user other than the news source feeds the news item into the Twitter } \\
\text { network, usually by using a 'Tweet this' button included on the source's } \\
\text { webpage containing the item. Such buttons offer an interface in which a } \\
\text { default text is given (usually the beginning of the title and a shortened } \\
\text { URL), which may be edited. }\end{array}$ \\
\hline $\begin{array}{l}\text { Content spread } \\
\text { by user }\end{array}$ & $\begin{array}{l}\text { The tweeted news item or a comment on the item (i.e., content that is } \\
\text { already in the Twitter network) is retweeted. We differentiate between } \\
\text { source content spread by users and user content spread by users. }\end{array}$ \\
\hline $\begin{array}{l}\text { Other source } \\
\text { content }\end{array}$ & The news source tweets without referring to a specific article (URL). \\
\hline $\begin{array}{l}\text { Other user } \\
\text { content }\end{array}$ & $\begin{array}{l}\text { Another user tweets about the source without referring to a specific } \\
\text { article (URL). }\end{array}$ \\
\hline
\end{tabular}

We classify, by means of an automatic script, actual tweets as instances of the types of tweets shown in Figure 1 by means of the navigational and communicational metadata (URLs and user mentions, respectively) and the tweets' contents. The metadata are either obtained explicitly from the Twitter API or parsed out

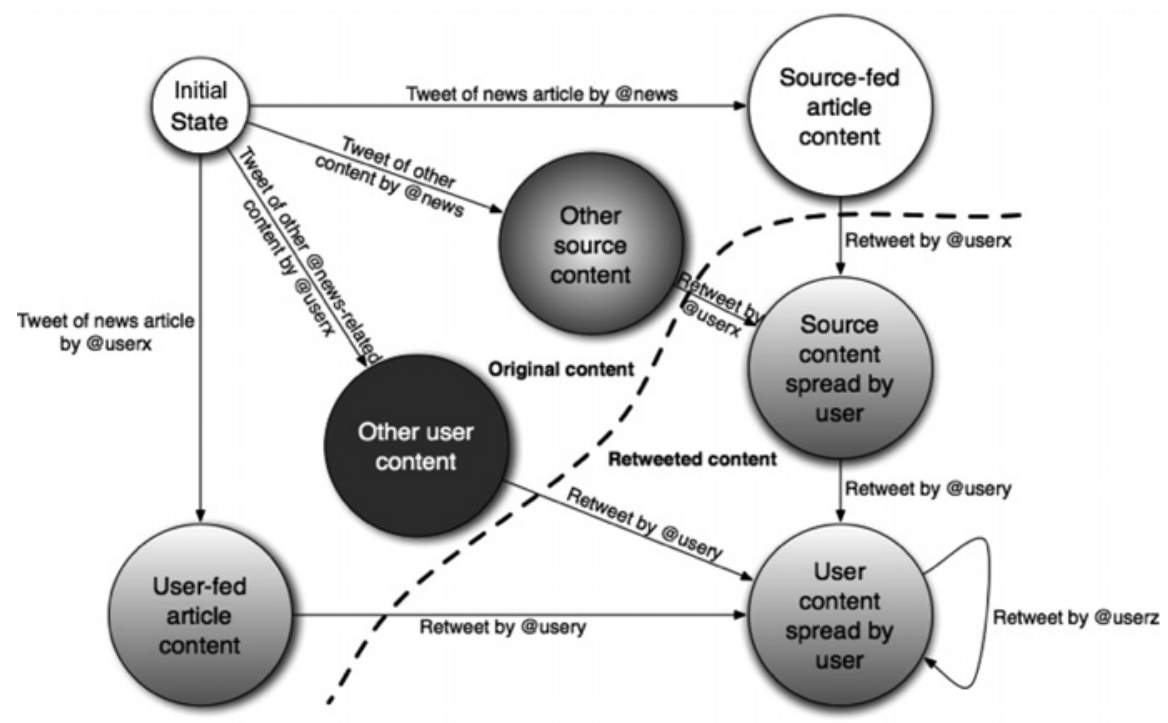

Figure 1: The information diffusion workflow of news in Twitter ('white' refers to no UGC while 'black' refers to UGC only). 
Girls expose FIFA weak point http://bit.ly/dxNzaP ------ QUOTED -------I----NavigMD----

\#Serbia expressing more interest in \#Russia http://t.co/DRvWaW19

- - - - - - QUOTED (hashtags inserted) - - - - - - |- - - - NavigMD- - - - -

RT @v: Analysts: \#Kosovo partition unrealistic (http:...) \#Serbia CommMD |----- QUOTED (hashtags inserted)----- - | NavigMD |-UGC (v)-

Hang the bastards RT @News24: 3 rhino poaching suspects held http:... ----UGC (v)-----|--COMmMD---|-------- QUOTED--------|NavigMD My latest piece: Cyprus celebrates independence with no solution in sight \#Cyprus \#Turkey \#UN http://t.co/C7aOfHPV

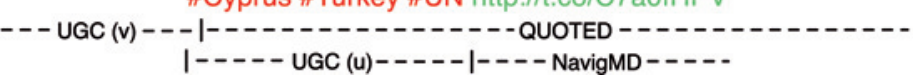

Figure 2: Examples of parsed tweets; italics show retweeted content.

of the text with regular expressions. Metadata on the retweet can serve to identify the original user and the full text of the original tweet. In the absence of retweetrelated metadata, the text is parsed as follows: If the tweet matches the string "string1 RT userx string2", then it is assumed that (a) it is a retweet, (b) the original user is userx, and (c) the original tweet is string2. URLs were extracted from the metadata or, with the help of regular expressions, from the tweet text.

Our parsing method measures the length of user-generated and of quoted content and can express these lengths (number of characters) also as proportions of the total length. Quotations are classified and measured by whether they are verbatim parts of the referred-to web pages or their titles, whether they are less straightforward excerpts (such as part of the title plus part of the first sentence), and by how similar they are to the title of the web page. In addition, we measure the intensity with which tweets categorize content by hashtagging terms (\#) and the intensity with which tweets are used for communication that is more specific than broadcasting, by user mentions (@). Some example tweets annotated by our parsing method are shown in Figure 2.

The background knowledge provided by social science research concerning media logic and communication patterns of the different platforms proved indispensable in fine-tuning this parsing model and the classification algorithms that were used.

Comparing the frequencies of the types can inform the analyst about the relative roles of individual Twitter users as multipliers of mainstream news media. Comparing the categories 'user-fed article content' versus 'content spread by users' can help infer statements about the attraction of external websites versus utterances already within the Twitter network, and the behavior of users active within these categories. Finally, tracing sequences and properties of the six categories can provide insights into the dynamics of reading, filtering, and discursively enriching news with the help of Twitter. 


\section{Data and data gathering (steps 2 and 3)}

According to Gartner Research analyst Doug Laney, a pioneer in the field of data warehousing, big data can be characterized based on three dimensions ${ }^{10}$ (also known as the ' $3 \mathrm{Vs}$ '): volume, velocity, and variety. Volume indicates the size of the data set that needs to be processed. The speed at which data comes in and the time needed to process it are denoted by the data velocity. Whereas earlier, data was processed in batch mode, now streaming data arrives continuously, which requires (near-)real time processing in order to be useful. The data variety refers to the differences in data formats. Data can be structured or unstructured, and one has less control over the format of incoming data. Recently, Gartner Research updated its definition and added a fourth ' $\mathrm{V}$ ' to this list, namely veracity. This denotes the integrity of the data and the ability of humans to trust it and use it to make decisions confidently. It may be clear that the latter is highly important for communication research and journalism studies.

Different techniques are used to collect tweets, ranging from open source infoveillance software (e.g., infovigil, see Chew and Eysenbach, 2010) to indexing programs (see e.g., Thelwall, Buckley, and Paltoglou, 2011), third-party websites like Twitalyzer and Topsy, and RSS-based plug-ins (e.g., Twitterpad). Other researchers have collected tweets using Twitter's own search engine (e.g., Sullivan et al., 2012). Some of these techniques are difficult to set up, and others are expensive or opaque about the way they collect the tweets. There are some researchers who collect tweets with a script that searches for tweets that meet specific criteria (such as a keyword, hashtag or location). Most of these studies (e.g., Lovejoy, Waters, and Saxton, 2012) simply mention that they used a selfwritten script without elaborating on how the script and the Twitter API actually work. Therefore, we will focus on the mechanism behind the Twitter API as a free, transparent and easily set up method for collecting tweets.

First of all, a distinction has to be made between the two possible options by which Twitter provides access to the collection of tweets. On the one hand, there is the commercial Twitter Firehose, a service that provides access to tweets in near real-time and guarantees the delivery of $100 \%$ of the tweets that match a user's search criteria. Access to the Twitter Firehose is offered by a number of Twitter-certified data resellers. ${ }^{11}$ On the other hand, there is the free

10 http://blogs.gartner.com/doug-laney/files/2012/01/ad949-3D-Data-ManagementControlling-Data-Volume-Velocity-and-Variety.pdf

$11 \mathrm{https} / / /$ dev.twitter.com/programs/twitter-certified-products/products 
Streaming API ${ }^{12}$ that offers low-latency access to a $1 \%$ sample of Twitter's global stream of tweet data. Comprehensive overviews of how to gather tweets with the Streaming API and perform (basic) analyses on the resulting dataset can be found in Bruns and Liang (2012) and Kumar, Morstatter, and Huan (2013).

This distinction is very important from a research perspective, as shown by Morstatter, Pfeffer, Liu, and Carley (2013). In a comparison of data from Twitter's free, but limited, Streaming API and the unlimited, but fee-based, Twitter Firehose, they investigated whether the sampled data was a valid representation of the overall activity on Twitter. They conclude that the results of using the Streaming API strongly depend on the intended coverage and type of analysis. For example, concerning network level measures, their analysis revealed a correlation between network centrality indexes and the proportion of data covered by the Streaming API. Therefore, before starting the (automated) analysis of a large set of tweets, one needs to choose the API appropriately.

A detailed description of the data gathering for the case study, along with a set of intuitive code snippets and links to free software packages that we offer for reuse by other researchers, can be found online.

\section{Example study: The TwiNe tool and a case (steps 4-6)}

Our system, TwiNeR (Twitter News Reading) consists of

a) parsing routines that data-mine tweets by automatically classifying and scoring them according to the model explained in the previous subsection, and

b) an interactive visualization user interface that allows the analyst to inspect tweet corpora.

In order to analyze the results from the previous data mining step, we need to go one step further than mere visualization approaches, since the researcher needs an efficient way to interact with the resulting data. This is provided by techniques based on visual data mining or visual analytics (Simoff, Böhlen, and Mazeika, 2008). As the name indicates, the latter offers a set of visual analysis tools and techniques (for overviews, see Keim, Kohlhammer, Ellis, and Mansmann, 2010; Stahl, Gabrys, Gaber, and Berendsen, 2013). In contrast to

12 https://dev.twitter.com/docs/streaming-apis 
information visualization, where the data is inspected as is, visual data mining focuses on analyzing and inspecting data that has been preprocessed by a data mining tool, and allows interaction with the data mining model. As outlined by Keim, Mansmann, Schneidewind, Thomas, and Ziegler (2008), the combination of these tools and the analytic capabilities of the human brain help improve the scalability and accuracy of the discovery of patterns within large and complex data sets. The authors summarize the process in the following Visual Analytics mantra: 'Analyze first - Show the important - Zoom, filter and analyze further - Give details on demand'. An important aspect in this process is the interactivity of the visualization, so that it can be dynamically optimized for the user, use case and situation it is used in.

Our system, TwiNeR, visualizes large-scale behavioral patterns in Twitter news-spreading, thereby helping the analyst to 'dig deeper' into the quantitative results by looking at individual tweets and distributions of behaviors. The tool rests on (a) a model of prototypical activities related to news and news sources and (b) a language-independent data-mining method for classifying tweets into these activity categories based on information extraction, and for automatically scoring their degree of content quoting, spreading and addition.

For the analysis, we used data and information relating to the 18 news sources from across all continents that are shown in Table 2. Already at this

Table 2: The news sources used.

\begin{tabular}{lll}
\hline Source & Country/Region & Search term \\
\hline Al Jazeera English & Qatar & AJ English \\
Asia Times Online & Thailand & Asia Times Online \\
BBC News (UK) & UK & BBC news \\
CNN & USA & CNN \\
Daily Nation & Kenya & Daily Nation \\
Ghana News & Ghana & FreshNEws_Ghana \\
MercoPress & Uruguay & MercoPressNews \\
News24 & South Africa & News24 \\
setimes & Southeast Europe & setimes \\
SPIEGEL English & Germany & Spiegel_english \\
The Australian & Australia & Australian \\
The Daily Maverick & South Africa & Dailymaverick \\
The Guardian & UK & Guardian \\
The Herald Sun & Australia & The Herald Sun \\
The New York Times & USA & NY Times \\
The Washington Post & USA & Washington Post \\
Thomson Reuters & USA & Thomson Reuters \\
Times of India & India & Times of India \\
\hline
\end{tabular}


point, the interdisciplinary approach bore fruit, as it allowed us to select a data set that was sufficiently large for automatic analysis, yet consisted of a representative set of news sources, substantiated by insights from social science research. This also allowed us to draw more solid conclusions. We collected tweets from and about these sources using the Twitter Search API ${ }^{13}$ and the queries shown in the table.

\subsection{Global analyses and comparisons between sources}

Figure 3 shows an example analysis of tweets by base type, comparing the UKbased BBC News against the American CNN. The analysis shows percentages of 9850 to 9935 tweets collected during the period October 3-4, 2012. It uses standard pie charts to focus on a baseline distribution that focuses on the 'flat' taxonomy of Table 1. It illustrates, among other things, the large divergence between the behavior of those users of the two sources who first introduce source-related content into Twitter: Whereas CNN users tend to feed article content (user-fed article content), BBC News users comment much more about the source, without direct references to a specific article (other user content).

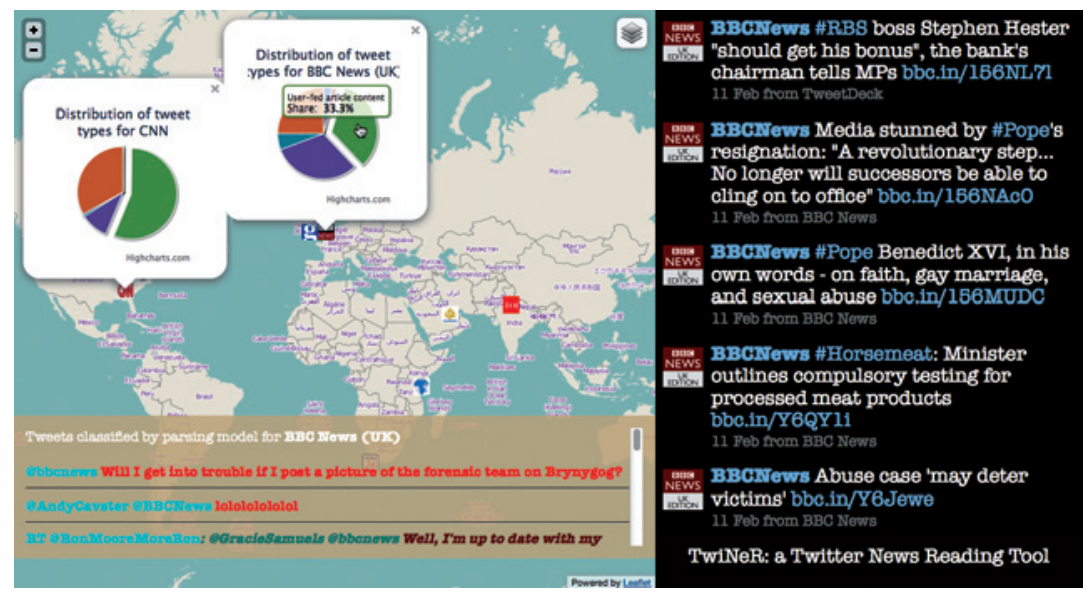

Figure 3: Comparing the distribution of tweet types across news sources.

$13 \mathrm{https} / /$ dev.twitter.com/docs/api/1.1/get/search/tweets 


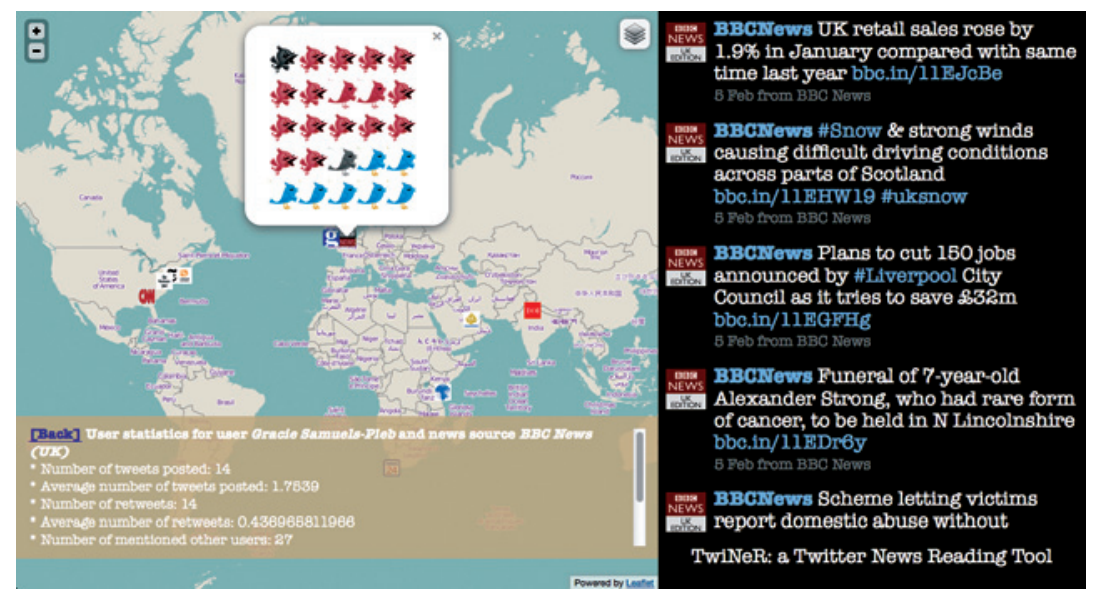

Figure 4: Drilling down into the analysis of tweet types for a news source (top left); analyzing tweets and users (bottom left).

\subsection{Drill-down analyses by source}

Figure 4 shows an example analysis that drills down further into the findings of Figure 3. First, types are now visualized with different dimensions. Color indicates the base type (dark grey: source-fed content; pink: user-fed content; light grey: source content spread by user; blue: user content spread by user). 'Talkative' birds represent tweets with significant amounts of user-generated content (for this figure: at least ten characters), whereas 'silent' birds represent tweets with only or predominantly quoted content. Finally, birds with pointing feathers represent tweets with URLs, and birds without pointing feathers represent tweets without URLs. ${ }^{14}$

\subsection{Text- and user-centric analyses}

Figure 4 also shows further drill-down possibilities for the analyst, allowing for a more user-centric investigation: Highlighting a bird will open a window that shows the tweets in this category in full text, along with their authors (bottom left). The colors of the text are the result of applying our parsing model to the tweet records collected from the Twitter API. Based on the texts of the tweet

14 Every bird represents 500 tweets; counts of categories were rounded to avoid having to show bird fragments. 
and (if applicable) the referred-to web page, as well as on metadata returned by the API, we parse the text into (a) content that is quoted from the web page, that is, from a news article (shown in blue); (b) URLs and user mentions as 'navigational' or 'communicational' metadata (shown in turquoise), and (c) user-generated content over and above the quoted content (shown in red). Finally, (d) retweeted content is shown in italics (with the blue, turquoise and slightly darker red when retweeted). The URLs allow the analyst to open the referred-to web pages for comparison with the tweet. Finally, users can be selected, and statistics shown (bottom right).

The visualization gives an interactive summary of some typical findings about users and tweets obtained in the data analysis step. First, much user content that contains a URL has the format of a short comment that precedes the retweeted content, which in turn often consists of only content lifted off the web page automatically, together with a pointer to the page. Second, this pattern can be found across natural languages (our parser is language-independent). Third, this type of tweeting is only rarely associated with selective communication operations: Further user mentions (beyond the mention of the author of the retweeted content after the 'RT') are added only rarely. Also, little activity is shown in terms of categorizing the content by adding hashtags, and the hashtags that do get added are often cumbersome and not re-used. Fourth, users in this category tend not to be 'opinion leaders' - in contrast, we find other users who (a) tweet a lot more (up to several hundreds of tweets in our 2-day sample) and (b) get retweeted often (again, up to hundreds of times). Fifth, adding one's own comments is minority behavior among the retweeters (there are many more 'silent' blue birds than 'talkative' ones), whereas users who first feed content into the Twitter network tend to be much more outspoken (witness the much larger proportion of 'talkative' pink birds).

Figure 5 (top) shows a breakdown by news source and tweet category, focusing on the actors and retweeted/new content dimensions. It illustrates that user feeding of article content into Twitter dominates activity across sources. Some sources have a high level of activity in terms of users referring to the source without spreading an article ('other user content') and, to a lesser extent, also in terms of the source tweeting without spreading an article ('other source content').

Figure 5 (bottom) investigates this behavior as one key aspect of content, with some aggregation over categories for better visibility. It analyzes the numbers above with respect to whether they contain a URL, thus illustrating the importance of referencing URLs in news-related tweets. The source's own feeding behavior is of course dominated by tweets with URLs, but not as exclusively as one might have expected. For most sources, URL-containing tweets dominate 

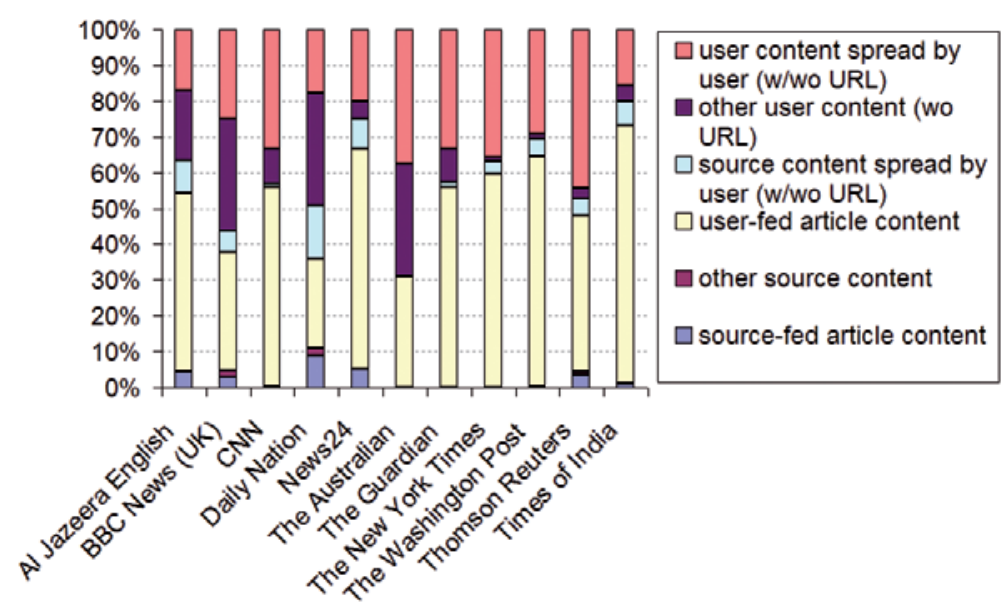

user (w/wo URL)
other user content (wo URL)

source content spread by user (w/wo URL)

$\square$ user-fed article content

$\square$ other source content

$\square$ source-fed article content
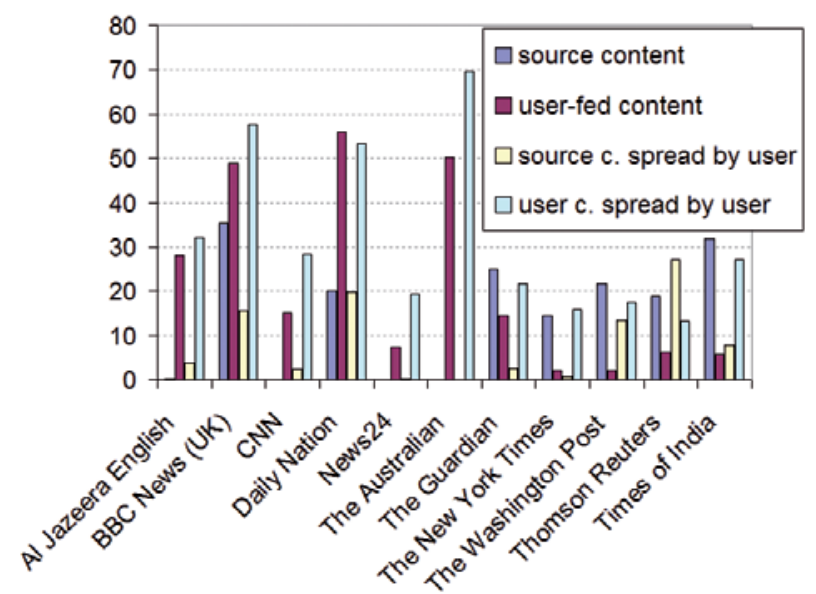

Figure 5: Percentages of tweets per category (top) and tweets without URLs (bottom).

the behavior of users who tweet about this source: For five sources, there are fewer than $10 \%$ without URL, for a further three, there are below $30 \%$. In three sources, however, half or more of user content does not contain a URL. The diagram also shows that source content loses its importance along the information diffusion cycle - in all but one case, retweeted tweets are more often URLfree than originally fed tweets. This could be a selection bias (tweets without a URL get retweeted more often) or the effect of content of the retweeted tweet being supplanted by new comments from the now-active user. To explain these different styles of tweeting news content of the sources and the corresponding 
retweeting behavior of the users, the domain expertise from journalism and media studies proved to be indispensable. For example, instead of just retweeting the article's title, some sources reformulate the content in a more appealing way in order to convince the user to click through to the source's website (the so-called clickbait).

Based on this quantitative analysis provided by TwiNer on the basis of two days of Tweet gathering, we tentatively conclude that users play a much larger role than the sources themselves in spreading news-related content. Within this group, the most content-shaping activity occurs at the moment when a user first feeds news content into Twitter: These tweets refer to original content of the (presumably trusted) news sources, but they also add their own comments, categorize material by adding hashtags, and drive communication by inserting user mentions. Such activity is far less pronounced in later stages of the information-diffusion workflow, where retweeting appears to be dominated by repetition without comments, categorizations, or other additions. Retweeters also more often refer to tweets without URL references in them. Thus, they less often give their readers the chance to delve deeper into a topic with the help of a URL.

Taken together, such behavior may be interpreted as indicating that Twitter is seldom used as a forum for critically discussing mainstream news content for discussion cannot stop after the first comment has been made. Selection alone (the decision what to retweet and what not) is not enough for critical news reception. In addition, if only a few first-feeders tweet a lot (as indicated by the Zipf distribution), some Twitter users may become the new gatekeepers of the news universe.

\section{Discussion and outlook}

We proposed data mining, the computational process of discovering patterns (i.e., knowledge) in large data sets, as a solution for the challenges that arise from analyzing big data. Our aim was to empower the communication scholar to extract knowledge from the new knowledge sources we are faced with today, while driving research in computer science based on the new research questions that originate from this collaboration. We discussed the analysis of big data in a communication-science research environment by means of an adaptation of the standard data mining process model, and illustrated the different steps by means of a case study and a software tool.

The first step, turning a communications-research question into a computation-research question was discussed by means of a case study, whereby we 
investigated how Twitter can be used to study news reading behavior. The characteristics and the gathering of big data were discussed on a more general level.

The subsequent steps of the analysis were presented by means of TwiNeR, a software tool that analyzes tweet content using an advanced language modeling approach for classifying tweets into prototypical activities related to news and news sources on Twitter. The tool is able to score tweets based on their degree of content quoting, spreading and addition with high accuracy in a language-independent way. Using the presented workflow of tweet types, distribution of these types and subtypes can be investigated and compared across news sources, so as to gain insight in the authoring style, behavior, and influence of individual users. In future work, we will remedy the syntactic and semantic limitations of TwiNer and explore further scenarios of application of the automated tool in the news domain and, by extension, in other domains in which outside content is referenced.

Both during the domain and data understanding phase, as well as during the sense-making and presentation of the results, the interdisciplinary approach resulted in a number of challenges originating from the methodological differences in both domains, yet also proved to be an added value.

These methodological differences can largely be explained by the scale at which a particular phenomenon is studied in both fields. While in social sciences, the goal is to study a particular phenomenon in detail (focus on depth), computer scientists aim at extracting patterns from the analysis of large amounts of information (focus on breadth). First of all, this became apparent during the data selection phase. The main challenge was to select a representative sample that was large enough to be analyzed automatically, yet fitted the methodological constraints posed by social science research. By combining both approaches, this allowed us to select a focused, yet sufficiently large sample, which allowed us to draw more solid conclusions. Also during the data analysis, the insights and domain knowledge of social media, and, more specifically, the media logic of the different platforms, news actors and communication patterns, proved helpful in more accurately tuning the language modeling and classification algorithms that were used in this study. In order to tackle the analysis in a principled way, we started by drafting the information diffusion workflow of news on Twitter as presented. As in computer science, we started this in a data-driven way, based on a subsample of tweets; due to the interdisciplinary approach this could now be complemented by the theoretical expertise concerning the different news actors and their interactions from journalism and media studies. An interactive approach with multiple iterations allowed us to come to a more complete, consolidated version as presented in Figure 1, which 
is both theoretically grounded as well as supported by the data. Finally, also during the validation phase, both domains proved to be instrumental to one another. The insights from journalism and media studies concerning the communication flow between the different news actors led to a deeper, more adequate interpretation of the obtained results than would have been possible when solely using automated data-mining methods (e.g., regarding the rationale of the news sources to include URLs or reformulate the article title in the tweet). On the other hand, the fact that the analysis could be automated to a certain extent resulted in a broader set of insights from a social sciences perspective.

The outcome of this study thus illustrated that both domains can indeed be instrumental to one another and even methodologically enriching. The results obtained provide a bridge between computer science, journalism studies (i.e., the study of news dynamics) and communication research (message analysis, gate-keeping research, and audience studies) in the so-called 'mixed media' domain, an area that is characterized both by fragmented audiences and the production of news by individuals rather than news professionals exclusively. When fueled by the mining of big data, the ability or lack thereof of newer technologies to foster a healthy democratic dialogue in society or issue plurality can be analyzed with considerably greater depth and breadth than when merely using the standard social sciences techniques such as content or discourse analysis combined with survey research. Thanks to automated analysis and through the identification of issue, source or frame correspondence or discrepancies, the interconnectedness between online networks of users and their degree of homophily or heterogeneity can be more robustly determined.

\section{References}

Allan, J. 2002. Introduction to topic detection and tracking. In Topic detection and tracking (pp. 1-16). New York: Springer US.

Allan, S. 2013. Citizen witnessing: Revisioning journalism in times of crisis. Cambridge: Polity Press.

Armstrong, C. J., \& Gao, F. 2011. Gender, Twitter and news content. An examination across platforms and coverage areas. Journalism Studies 12(4). 490-505.

Benhardus, J., \& Kalita, J. 2013. Streaming trend detection in Twitter. International Journal of Web Based Communities 9(1). 122-139.

Berendt, B. 2010. Text mining for news and blogs analysis. In Encyclopedia of Machine Learning (pp. 968-972). New York: Springer US. 
Bollen, J., Mao, H., \& Pepe, A. 2011. Modeling public mood and emotion: Twitter sentiment and socio-economic phenomena. Proceedings of the Fifth International AAAl Conference on Weblogs and Social Media (ICWSM 2011). 17-21 July, Barcelona, Spain.

boyd, d., \& Crawford, K. 2012. Critical questions for big data. Information, Communication \& Society 15(5). 662-679.

boyd, d., Golder, S., \& Lotan, G. 2010. Tweet, tweet, retweet: Conversational aspects of retweeting on twitter. Proceedings of HICSS-42, Persistent Conversation Track. Kauai, HI: IEEE Computer Society, 5-8 January.

Bruns, A., \& Burgess, J. 2012. Researching news discussion on Twitter: New methodologies. Journalism Studies 13(5-6). 801-814.

Bruns, A., \& Liang, Y. E. 2012. Tools and methods for capturing Twitter data during natural disasters. First Monday 17(4).

Cha et al. 2010. Measuring influence in Twitter: The million follower fallacy. Proceedings of the Fourth International Conference on Weblogs and Social Media (ICWSM 2010). 23-26 May, 2010, Washington, DC, USA.

Chew, C., \& Eysenbach, G. 2010. Pandemics in the age of Twitter: Content analysis of Tweets during the 2009 H1N1 outbreak. PloS one, 5(11), e14118.

Diakopoulos, N., De Choudhury, M., \& Naaman, M. 2012. Finding and assessing social media information sources in the context of journalism. Conference on Human Factors in Computing Systems (CHI). May, 2012.

Easley, D., \& Kleinberg, J. 2010. Networks, crowds, and markets (vol. 8). Cambridge: Cambridge University Press.

Fayyad, U., Piatetsky-Shapiro, G., \& Smyth, P. 1996. From data mining to knowledge discovery in databases. Al magazine 17(3). 37.

Gamon, M., Basu, S., Belenko, D., Fisher, D., Hurst, M., \& König, A. C. 2008. BLEWS: Using blogs to provide context for news articles. Proceedings of the International Conference on Weblogs and Social Media (ICWSM).

Glasgow, K., \& Fink, C. 2013. Hashtag lifespan and social networks during the London riots. In A. M. Greenberg, W. G. Kennedy \& N. D. Bos (Eds.), Social computing, behavioralcultural modeling and prediction. Berlin, Heidelberg: Springer.

Hansen, L. K., Arvidsson, A., Nielsen, F. Å., Colleoni, E., \& Etter, M. 2011. Good friends, bad news-affect and virality in Twitter. In J. J. Park, L. T. Yang \& C. Lee (Eds.), Future information technology. Berlin, Heidelberg: Springer.

Honey, C., \& Herring, S. C. 2009. Beyond microblogging: Conversation and collaboration via Twitter. In System Sciences, 2009. HICSS '09, 42 ${ }^{\text {nd }}$ Hawaii International Conference on System Sciences, January 5-8.

Hu, M., Liu, S., Wei, F., Wu, Y., Stasko, J., \& Ma, K. L. 2012. Breaking news on Twitter. Proceedings of ACM CHI 2012 (pp. 2751-2754). Austin, May 2012.

Jothi, P. S., Neelamalar, M., \& Prasad, R. S. 2011. Analysis of social networking sites: A study on effective communication strategy in developing brand communication. Journal of Media and Communication Studies 3(7). 234-242.

Karpf, D. 2012. Social science research methods in internet time. Information, Communication \& Society 15(5). 639-661.

Keim, D. A., Kohlhammer, J., Ellis, G., \& Mansmann, F. (Eds.). 2010. Mastering the information age - Solving problems with visual analytics. Goslar: Eurographics Association.

Keim, D. A., Mansmann, F., Schneidewind, J., Thomas, J., \& Ziegler, H. 2008. Visual analytics: Scope and challenges (pp. 76-90). Berlin: Springer. 
Kumar, S., Morstatter, F., \& Huan L. 2013. Twitter data analytics. New York: Springer.

Kwak, H., Lee, C., Park, H., \& Moon, S. 2010. What is Twitter, a social network or a news media? Proceedings of the $19^{\text {th }}$ International World Wide Web (WWW) Conference, April 26-30, Raleigh, NC.

Lerman, K., \& Ghosh, R. 2010. Information contagion: An empirical study of the spread of news on Digg and Twitter social networks. Proceedings of the $4^{\text {th }}$ International Conference on Weblogs and Social Media (ICWSM) (pp. 90-97).

Leskovec, J., Backstrom, L., \& Kleinberg, J. 2009. Meme-tracking and the dynamics of the news cycle. Proceedings of the $15^{\text {th }}$ ACM SIGKDD international conference on knowledge discovery and data mining (pp. 497-506).

Lovejoy, K., Waters, R. D., \& Saxton, G. D. 2012. Engaging stakeholders through Twitter: How nonprofit organizations are getting more out of 140 characters or less. Public Relations Review 38(2). 313-318.

Mei, Q., \& Zhai, C. 2005. Discovering evolutionary theme patterns from text: An exploration of temporal text mining. Proceedings of the $11^{\text {th }}$ ACM SIGKDD international conference on knowledge discovery in data mining (pp. 198-207).

Meraz, S. 2011a. The fight for how to think: Traditional media, social networks, and issue interpretation. Journalism: Theory, Practice, and Criticism 12(1). 107-127.

Meraz, S. 2011b. Using time series analysis to measure intermedia agenda-setting influence in traditional media and political blog networks. Journalism \& Mass Communication Quarterly 88(1). 176-194.

Morstatter, F., Pfeffer, J., Liu, H., \& Carley, K. M. 2013. Is the sample good enough? comparing data from Twitter's Streaming API with Twitter's Firehose. Proceedings of the International AAAI Conference on Weblogs and Social Media (ICWSM) (pp. 400-408).

Naisbitt, J. 1982. Megatrends. New York: Warner.

Papacharissi, Z., \& de Fatima Oliveira, M. 2012. Affective news and networked publics: The rhythms of news storytelling on \#Egypt. Journal of Communication 62(2). 266-282.

Qazvinian, V., Rosengren, E., Radev, D. R., \& Mei, Q. 2011. Rumor has it: Identifying misinformation in microblogs. Proceedings of the 2011 Conference on Empirical Methods in Natural Language Processing (pp. 1589-1599).

Simoff, S., Böhlen, M. H., \& Mazeika, A. (Eds.). 2008. Visual data mining: Theory, techniques and tools for visual analytics (vol. 4404). Berlin: Springer.

Stahl, F., Gabrys, B., Gaber, M., \& Berendsen, M. 2013. An overview on interactive visual data mining techniques for knowledge discovery. Wiley Interdisciplinary Reviews: Data Mining and Knowledge Discovery, 3(4), 239-256.

Subašic, I., \& Berendt, B. 2010. From bursty patterns to bursty facts: The effectiveness of temporal text mining for news. Proceedings of the $19^{\text {th }}$ European Conference on Artificial Intelligence, Lisbon, August 16-20 (pp. 517-522).

Subašić, I., \& Berendt, B. 2011. Peddling or creating? Investigating the role of Twitter in news reporting. In Advances in Information Retrieval (pp. 207-213). Berlin: Springer.

Sullivan, S. J., Schneiders, A. G., Cheang, C. W., Kitto, E., Lee, H., Redhead, J., \& McCrory, P. R. 2012. 'What's happening?' A content analysis of concussion-related traffic on Twitter. British Journal of Sports Medicine 46(4).

Szomszor, M., Kostkova, P., \& St Louis, C. 2011. Twitter informatics: Tracking and understanding public reaction during the 2009 swine flu pandemic. Proceedings of the 2011 IEEE/WIC/ACM International Conference on Web Intelligence, WI. 320-323.

Terpstra, T., de Vries, A., Stronkman, R., \& Paradies, G. L. 2012. Towards a realtime Twitter analysis during crises for operational crisis management. Proceedings of the $9^{\text {th }}$ 
International Conference on Information Systems for Crisis Response and Management, 22-25 April, Vancouver, BC.

Thelwall, M., Buckley, K., \& Paltoglou, G. 2011. Sentiment in Twitter events. Journal of the American Society for Information Science and Technology 62(2). 406-418.

Tufekci, Z., \& Wilson, C. 2012. Social media and the decision to participate in political protest: Observations from Tahrir Square. Journal of Communication 62(2). 363-379

Vergeer, M., \& Hermans, L. 2013. Campaigning on Twitter: Microblogging and online social networking as campaign tools in the 2010 general elections in the Netherlands. Journal of Computer-Mediated Communication 18(4). 399-419.

Witten, I. H., Frank, E., \& Hall, M. A. 2011. Data mining: Practical machine learning tools and techniques. Burlington: Elsevier. 